\title{
Quelques observations et réflexions sur les projets éducatifs des collèges québécois pour garçons à partir d'un exemple : Sainte-Anne de La Pocatière au $19^{e}$ siècle
}

\section{Christine Hudon}

\begin{abstract}
RÉSUMÉ
À partir d'un exemple, celui du Collège de Sainte-Anne de La Pocatière, cet article analyse les projets éducatifs développés par l'institution collégiale au $19^{\mathrm{e}}$ siècle afin de mettre en lumière les innovations qui s'y sont déployées. Dans un premier temps, nous nous arrêtons sur les objectifs poursuivis initialement par les promoteurs et sur les premières années d'existence de ce collège fondé en 1829. Cette étude de cas permet de montrer que l'institution, loin d'être arrimée à une tradition fixe et immuable, est marquée par le contexte sociopolitique et économique de son temps et qu'elle incorpore dans son enseignement les réflexions pédagogiques de son époque à propos de la méthode, de l'ordonnancement des matières et des finalités que l'on souhaite assigner à l'éducation.
\end{abstract}

\section{ABSTRACT*}

Based on an example, that of Sainte-Anne de La Pocatière College, this article analyzes the educational projects developed by the college during the 19th century in order to highlight the innovations that were implemented there. First of all, the objectives initially pursued by the promoters and those within the first years of existence of the college, founded in 1829, are examined. This case study demonstrates that the institution far from being bound to a fixed and unchanging tradition, is influenced by the socio-political and economic context of its time and incorporates in its teaching the pedagogical concerns of the period with relation to methods, organization of subjects and desired results attributed to education.

Dans l'historiographie de l'éducation, l'ouvrage de Claude Galarneau, Les collèges classiques au Canada français, occupe une place de premier plan. Publié il y a 30 ans, en réponse à une commande de l'Association des universités et collèges québécois, le livre s'intéresse au développement de l'institution des collèges classiques sur le territoire canadien et veille à cerner les caractéristiques de son corps enseignant, 
de sa clientèle, des cours et des programmes. L'étude entend montrer « comment le modèle culturel élaboré en Europe s'est implanté au Canada français et par quelles voies originales il a su se développer d'une façon aussi extraordinaire, de l'Atlantique aux Rocheuses ». ${ }^{1}$

Afin de remplir l'immense mandat qui lui avait été confié, Galarneau a dû faire des choix, résoudre des problèmes, l'un d'entre eux étant de définir et de circonscrire son objet d'étude. Ainsi a-t-il fait état, dans son analyse, de quelque 200 institutions, la majorité destinée aux garçons. Les noms utilisés pour les désigner étaient très variés, et l'auteur lui-même, dans son ouvrage, parle tantôt de collèges, tantôt de collèges-séminaires, tantôt de collèges classiques. Les contextes de fondation, les milieux d'implantation, le type de clientèles et de personnel différaient aussi d'un établissement à l'autre. Certains accueillaient une majorité de pensionnaires, d'autres étaient des externats. Les uns étaient localisés en ville et fréquentés par une clientèle à première vue plutôt aisée, d'autres étaient situés en milieu rural et recrutaient bon nombre d'enfants d'agriculteurs. Quelques collèges créés aux $19^{\mathrm{e}}$ et $20^{\mathrm{e}}$ siècles étaient dirigés par des communautés religieuses - les jésuites, par exemple — mais la plupart avaient une équipe enseignante constituée de prêtres séculiers et d'ecclésiastiques poursuivant leurs études théologiques.

Comme toute synthèse, celle de Galarneau a mis l'accent sur les similitudes. Elle a dégagé un certain nombre de caractéristiques générales, qui semblaient avoir peu varié dans le temps, l'auteur faisant en quelque sorte de la fidélité à la tradition le fil conducteur de son livre. Quelque 15 ans après le rapport Parent, il s'agissait finalement de mesurer l'écart entre le "nouvel humanisme " institué par la réforme, et l'ancien humanisme, l'humanisme classique ${ }^{2}$, héritage d'un Québec dont on célébrait désormais l'entrée dans la "modernité ". L'ouvrage a donc focalisé son attention sur l'importance du modèle jésuite, celui du cursus studiorum élaboré au $16^{\mathrm{e}}$ siècle, qui définit les programmes et les méthodes d'enseignement de l'humanisme chrétien fondés sur une étude assidue des maîtres de l'Antiquité profane et sacrée. Sans nier les adaptations survenues au cours des années et l'apparition de filières commerciales et industrielles, l'auteur ne les a guère analysées. Il a plutôt montré le poids du latin et de la rhétorique dans l'enseignement, le collège n'ayant " pas pour fonction d'innover, mais d'inculquer des vérités consacrées et des règles reconnues, de transmettre des savoirs tout en façonnant l'honnête homme et le catholique ». ${ }^{3}$ Il a également dépeint l'institution comme une véritable pépinière de prêtres : toutes périodes confondues, $40 \%$ à $45 \%$ des anciens élèves auraient opté pour le sacerdoce. ${ }^{4} \mathrm{Au}$ total, il estimait que le collège classique avait joué un rôle fondamental auprès des Canadiens français, celui de transmettre un riche héritage pluriséculaire.

Dans le cadre de cet article, nous voulons pour notre part revenir sur l'autre versant de cette histoire des collèges pour garçons, non pas celui de la tradition, mais celui de l'innovation. Plus précisément, nous souhaitons explorer ces " voies originales " que l'institution a empruntées. Ce besoin de questionner à nouveau les projets pédagogiques développés par les collèges a semblé de plus en plus manifeste à notre équipe, composée de Louise Bienvenue, d'Ollivier Hubert et de moi-même, au fur et à mesure que progressaient nos recherches sur les modèles masculins promus par 
l'institution collégiale avant 1960. Inspirés par Claude Galarneau, nous avions, dans un premier temps, limité notre analyse aux " collèges classiques » et tenté de voir quelles attitudes, quelles valeurs et quelles normes comportementales ceux-ci avaient proposées à la jeunesse québécoise. Peu à peu, cependant, s'est posé un problème bien concret : celui de distinguer les collèges " classiques " des autres établissements. Trois des quatre institutions retenues pour l'analyse à partir de la liste dressée dans Les collèges classiques au Canada français (les collèges de Nicolet, Sainte-Anne de La Pocatière, Saint-Hyacinthe et Sherbrooke) se présentaient en quelque sorte comme des modèles hybrides, qui ne correspondaient pas en tous points au modèle type du collège classique qui mettait l'accent sur les humanités. Un examen des registres et des autres livres d'élèves constituées par l'institution, et une lecture de la correspondance des supérieurs, directeurs d'élèves et des parents montraient également que ces établissements avaient évolué considérablement avec le temps, si bien que le collège des années 1950 se distinguait à maints égards — et souvent de manière très importante — de celui des années 1820 ou 1830 . Aussi, pour mieux comprendre le milieu de vie au sein duquel vivaient au quotidien les élèves, pour saisir la culture dans laquelle ils ont baigné et pour cerner les ambitions pédagogiques qu'avaient leurs maîtres, il nous a semblé nécessaire de revenir sur l'institution, sur ce lieu diversifié et complexe que furent les collèges classiques.

Dans cet article, nous porterons notre regard sur un seul établissement, le Collège de Sainte-Anne de La Pocatière. Ce changement d'échelle par rapport à la synthèse permettra de montrer que l'institution incorpore des influences diverses pendant les premières années de son existence. Ainsi l'histoire du collège est-elle à la fois marquée par les réflexions pédagogiques du temps à propos de la méthode, de l'ordonnancement des matières et des finalités que l'on souhaite assigner à l'éducation, par le contexte sociopolitique et par les caractéristiques socioéconomiques du milieu dans lequel il prend place. Sise sur la rive sud du fleuve, à environ 100 kilomètres de Québec, La Pocatière se trouve au cœur d'une région de colonisation relativement ancienne. ${ }^{5}$ Dans la première moitié $\mathrm{du} 19^{\mathrm{e}}$ siècle, cette région très massivement francophone et catholique a tous les traits d'un terroir plein. La saturation de l'espace seigneurial entraîne un solde migratoire négatif. Ce "monde d'agriculteurs " ${ }^{6}$, est constitué de familles bien établies et prospères et de producteurs aux revenus plus limités. Par ailleurs, on y retrouve aussi un petit groupe de notables qui se distinguent par leur fortune, leur culture et leur ascendant social. Tous ces facteurs auront une incidence sur le développement de l'établissement.

Comme l'ont fait récemment quelques auteurs ${ }^{7}$, nous souhaitons donc ici porter notre attention sur les projets éducatifs mis en place au $19^{\mathrm{e}}$ siècle. Dans un premier temps, nous chercherons à cerner les objectifs poursuivis initialement par les promoteurs et pour revenir sur les premières années d'existence du collège. Par la suite, nous nous arrêterons sur une de ses particularités, son cours commercial, inauguré en 1842. À terme, une telle démarche fondée essentiellement sur l'analyse des programmes, des prospectus, de la correspondance et des listes d'élèves du Collège de Sainte-Anne, nous conduira à proposer quelques considérations sur la place du cours classique au sein des établissements collégiaux et sur l'évolution qu'ont connue ceux-ci. 


\section{Un plan d'éducation et un programme études originaux}

Le Collège de Sainte-Anne ouvre officiellement ses portes en 1829, à l'époque où d'autres maisons semblables voient le jour. Après Nicolet, Saint-Denis-sur-Richelieu, Saint-Hyacinthe, Saint-Roch, Sainte-Thérèse et Chambly, il s'agit, en fait, du septième collège fondé hors des villes de Québec et Montréal. Il est mis en place grâce aux efforts obstinés que déploie, à compter de 1820, son fondateur, le curé Painchaud de Sainte-Anne, qui bénéficie de l'appui de la notabilité locale. Les obstacles auxquels l'ecclésiastique est confronté sont essentiellement de deux ordres : l'évêque de Québec tarde à donner son accord au projet car il redoute que l'ouverture d'une nouvelle maison ne nuise aux autres établissements, en particulier au collège de Nicolet, créé en 1803. Par ailleurs, les paroisses voisines de Rivière-Ouelle et, surtout, de Kamouraska, caressent elles aussi le rêve d'ouvrir sur leur territoire une école dispensant un enseignement "secondaire ". Kamouraska mène d'ailleurs des démarches insistantes auprès des autorités diocésaines et de la Chambre d'Assemblée. Mû par la crainte que le projet essentiellement laïque de Kamouraska se concrétise et succombant aux pressions de Jérôme Demers, professeur au séminaire de Québec et ami de Painchaud, l'évêque Panet finit par donner son consentement à l'ouverture du collège de Sainte-Anne. ${ }^{8}$

Le curé Painchaud formule un programme d'études pour le moins original. Depuis plusieurs années, il s'intéresse de près à l'éducation. Déjà, en 1820, il a croisé le fer à ce sujet avec l'abbé Calonne, prêtre français exilé par la Révolution, qui dénonce les écoles lancastriennes ${ }^{9}$ et soutient, en citant Lamennais, que " la religion est l'unique éducation du peuple ". ${ }^{10}$ Sous le pseudonyme de Campagnard, Painchaud lui réplique dans la Gazette de Québec. Il y affirme l'urgence pour les Canadiens de savoir lire et écrire " eu égard à leur position politique » et, sans adhérer au projet libéral d'écoles financées par le gouvernement, il prône l'éducation comme moyen pour le peuple de participer à la vie politique et d'atteindre les « biens » et les " honneurs ». Le système d'études et le règlement mis en place à Sainte-Anne, en 1829, s'inscrivent tout à fait dans cet objectif, si cher à Painchaud, qui souhaite que son école forme des hommes capables de rendre " de grands services partout ". Dans un prospectus publié le $1^{\text {er }}$ août 1828 dans le journal le Canadien, il annonce les principes censés guider sa démarche enseignante. ${ }^{11}$ Le curé de Sainte-Anne propose un collège où " la différence de religion n'influera en rien sur l'admission ni le traitement des élèves ». À ceux-ci est garantie "la plus grande liberté religieuse possible sous un règlement catholique ». Ouvert à certains accommodements, le règlement initial s'éloigne de façon marquée de la règle quasi monacale des petits séminaires, comme, du reste, de la discipline assez stricte à laquelle ont souvent donné lieu les expériences d'enseignement mutuel. Si l'intention première n'exclut pas la formation d'une relève cléricale, toute l'organisation de la maison n'est cependant pas orientée vers cet objectif. Le règlement, en effet, n'impose pas d'uniforme et privilégie les remontrances et les punitions utiles et constructives, qui font réfléchir l'élève, aux châtiments corporels et aux punitions humiliantes. L'enseignement doit se faire selon les principes lancastriens, qui sont déjà appliquées au Bas-Canada dans les écoles soutenues par les Sociétés d'éducation 
de Québec, de Montréal et de Trois-Rivières, les écoles de Joseph-François Perreault et quelques écoles primaires issues des lois créant les écoles de l'Institution royale et de Syndics ${ }^{12}$, et encourager le développement des talents individuels. Le programme d'études vise à former un jeune homme "sachant un peu de tout ". Il s'écarte de la Ratio Studiorum, même s'il en reprend les principales matières : le français, le latin et, éventuellement, le grec, auquel s'ajoute l'anglais. "La rhétorique, la logique, la métaphysique, la physique [...] viendront par la suite, mais non dans l'ordre usité jusqu'à présent dans ce pays $"{ }^{13}$

Apprendre à " savoir vivre ", cultiver "l'art si précieux de conserver la santé et de la recouvrer après l'avoir perdue " : tel est l'un des axes majeurs du programme d'études publié par Painchaud en 1828. Grand lecteur et admirateur de Chateaubriand, le fondateur veut faire de sa maison un endroit sain, où l'élève pourra tirer partie de la « campagne romantique, ornée de pics, de montagnes, de rivières, de lacs " pour étudier l'histoire naturelle ou l'agriculture pendant ses jours de congé. À l'instar de Locke et de Rousseau, il entend former un " homme " qui n'est pas uniquement pétri de connaissances livresques, qui a aussi acquis un savoir pratique, utile, comme les Autochtones : "que savais-tu, pauvre enfant, disait si bien ce sauvage Abénaquis au jeune anglais adoptif, à qui il rendait sa liberté, qui savais-tu quand je t'ai adopté? - Rien ". ${ }^{14}$ Avec son collège, Painchaud souhaite faire œuvre de réformateur. Il s'agit « d'essayer un système raisonné et désiré par les amis de l'éducation ".

Deux personnes, l'abbé Painchaud et Julien Saillant, un instituteur du village, collaborèrent de près à la réflexion sur les principes éducatifs qui devaient marquer les débuts du collège. Il est cependant difficile de préciser l'influence qu'eut chacun d'eux sur l'élaboration de ce projet d'éducation. Les archives du Collège de SainteAnne conservent un plan d'éducation écrit de la main de Julien Saillant. ${ }^{15}$ Painchaud avait d'abord songé à lui pour la direction du collège, mais, pour des raisons qui restent obscures, l'homme quitte la paroisse avant même l'ouverture de la maison. Ce plan d'études met sur papier un certain nombre d'éléments qui seront repris dans le Prospectus de 1828, notamment en ce qui concerne le système lancastrien. Le document propose aussi une organisation des classes et des salles d'études différente de la disposition usuelle : les élèves sont assis en demi-cercle autour du maître, et non pas en rangées. Saillant est-il l'auteur de ce texte ? Wilfrid Lebon, dans son Histoire du collège de Sainte-Anne-De-La-Pocatière, répond à cette question par la négative : le brave instituteur aurait agi simplement à titre de scribe, commissionnaire et délégué de Painchaud auprès de $\mathrm{M}^{\text {gr }}$ Panet. Les idées ne venaient pas de lui. ${ }^{16} \mathrm{Il}$ est possible toutefois, et même probable, que l'expérience concrète de l'homme ait contribué à nourrir les réflexions pédagogiques du curé. À cet égard, il faut sans doute lire avec un scepticisme de bon aloi les affirmations de Lebon, fondées sur la mémoire institutionnelle et dont l'un des objectifs est de rappeler les efforts du curé fondateur et ses multiples sacrifices pour l'érection d'une maison d'éducation.

L'autre figure importante qui marqua les débuts du collège et inspira sa pédagogie initiale fut Étienne Chartier. Avocat de formation, il étudie au Séminaire de Québec pour devenir prêtre, quand le curé de Sainte-Anne l'approche pour en faire le directeur de sa maison. L'école élémentaire qu'il a dirigée à L'Assomption, en 1825, lui a 
procuré une expérience concrète de l'enseignement auprès des jeunes ruraux. À la demande de Painchaud, Chartier rédige, à l'automne 1828, un mémoire sur l'éducation qu'il souhaite mettre en œuvre à Sainte-Anne. ${ }^{17}$ Comme certains membres de la bourgeoisie libérale de son époque, il critique la pédagogie jésuite et la discipline très stricte qui règne dans les collèges :

Parce que la Société des Jésuites a possédé dans son sein des hommes vraiment savans, on en infere que l'éducation que l'on recevait dans leurs collèges est la meilleure possible : Et comme nos collèges en ce pays où viennent des Jésuites ou sont calqués sur leur enseignement on regarde comme témérité damnable d'oser y trouver quelque chose à redire $\&$ et on croit fermer la bouche à quiconque ose faire la moindre critique, en lui demandant s'il se croit plus capable que les Jésuites $[\ldots] .^{18}$

Une réforme de l'enseignement lui semble donc nécessaire et urgente : «Du solide et du nouveau! Voilà l'engagement tacite que je prends envers le public, qui a les yeux fixés sur Ste Anne ». ${ }^{19}$ Le projet pédagogique ébauché par Chartier, s'il ne remet pas en question la nécessité d'enseigner les humanités, n’en témoigne pas moins d'une réflexion approfondie sur l'enseignement, fondée sur la lecture et la méditation de nombreux réformateurs clercs et laïques. Ainsi, puise-t-il aux idées de l'abbé Claude Fleury, pédagogue français du $17^{\mathrm{e}}$ siècle, soucieux de rendre l'instruction attrayante et stimulante pour l'enfant, et aux conceptions de Charles Rollin, en particulier celles du Traité des études, ce "livre phare " du début du $18^{\mathrm{e}}$ siècle, qui place la pédagogie dans le champ de la méthode " en laissant moins de place à la spéculation chrétienne $"{ }^{20}$ Le sensualisme de Condillac, philosophe des Lumières, et les propositions de Dumarsais pour simplifier et abréger l'enseignement du latin l'inspirent également. Enfin, son mémoire évoque les expériences du Père Girard, pédagogue suisse du tournant du $19^{\mathrm{e}}$ siècle, promoteur de l'enseignement mutuel.

À Sainte-Anne, Chartier souhaite repenser la discipline. Pour ce faire, il prône la mise en œuvre d'un système où les élèves choisissent leurs représentants et élisent un jury d'élèves chargé de sanctionner les fautifs. La Constitution britannique se trouve au cour de ses réflexions pédagogiques. À l'heure des conflits sociaux et politiques s'incarnant dans les luttes de l'Assemblée pour affirmer son pouvoir face au gouverneur, Chartier ambitionne en effet de faire œuvre d'éducation civique auprès de la jeunesse des campagnes. Non seulement veut-il briser la routine qui caractérise la vie quotidienne et les études dans les collèges de l'époque, il compte aussi repenser le collège : celui-ci doit s'adapter à "l'esprit du siècle » et enseigner « la connaissance du gouvernement politique » de la société :

Quelque surprenante \& extraordinaire que puisse paraître cette idée au premier coup d'œil, en réfléchissant un peu, on devrait s'apercevoir que la forme de l'éducation publique pour être bonne, devrait être adaptée à la forme du gouvernement sous lequel l'élève aura à vivre un jour ... Concluons donc que l'éducation est l'étude de la vie sociale \& qu'une maison d'éducation doit 
être la matrice du citoyen. Il doit y apprendre que si l'homme a des devoirs à remplir, il a aussi des droits qu'on doit respecter. L'on voit que je parle ici de l'homme destiné à vivre sous des institutions libérales (sous une constitution libre telle que la nôtre).

\section{$[.$.}

vous voulez donc, me dira-t-on en goguenardant, que votre règlement soit calqué sur la constitution d'Angleterre? Et pourquoi pas! Puisque l'élève de Ste Anne aura à vivre sous la constitution Britannique \& non pas à être régi par le Divan du Grand Seigneur. ${ }^{21}$

Ces propositions montrent bien qu'à Sainte-Anne, on n'applique pas un modèle tout prêt. Ainsi, à ses débuts, le plan d'études et l'organisation du collège n'obéissent pas au système extrêmement structuré, hiérarchisé, voire autoritaire, de la Ratio jésuite. Contrairement à ce qu'a pu écrire Fernand Ouellet, le règlement initial de cet établissement ne reflète nullement "une volonté bien arrêtée d'isoler les écoliers du monde afin de les soustraire à ses influences $" .{ }^{22}$ Les premières années, le tiers environ des élèves sont d'ailleurs externes, comme ce semble être aussi le cas dans d'autres collèges. $^{23}$

La volonté d'expérimentation, de nouveauté et d'ajustements aux réalités politiques et sociales du temps qui le caractérise suscite l'intérêt. En 1831, l'avocat ComeSéraphin Cherrier louange les sacrifices de Painchaud pour " procurer à ses jeunes compatriotes une éducation religieuse et libérale tout ensemble ${ }^{24}$ Toutefois, ces innovations ont également tôt fait de provoquer critiques et tensions. Au sein même de l'institution, l'équipe enseignante est secouée par des querelles. Le jour de la bénédiction, en septembre 1829, le directeur des études, Étienne Chartier, attire l'attention des journaux et des milieux politiques par ses déclarations controversées sur les Britanniques qu'il accuse d'imposer aux Canadiens le " mépris ", la " dégradation " et "l'esclavage politique ". ${ }^{25}$ Ce discours, qui s'inscrit dans une période des plus tendues marquée par les affrontements politiques et par le rappel, en 1828, du gouverneur Dalhousie, force le supérieur du collège, le curé Painchaud, à intervenir auprès du gouverneur Kempt pour tenter de calmer le jeu. ${ }^{26}$ Dès lors, le supérieur et les cinq jeunes ecclésiastiques qui enseignent au collège au cours de la première année regardent avec méfiance les agissements du directeur des études. Par ailleurs, des rumeurs circulent à son sujet et des accusations de débauche et de sodomie viennent aux oreilles de l'évêque qui le retire de ses fonctions au terme d'une année. ${ }^{27}$ Son successeur, Louis Proulx, arrivé en octobre 1830, se désole du piètre état des lieux et des élèves qu’il juge " mal élevés et ignorants ». Attribuant ces défauts aux " effets funestes de leur trop grande liberté ", il entreprend un programme de réformes. ${ }^{28}$ Une clôture est posée, l'uniforme est prescrit, le droit de fumer est aboli, la messe devient obligatoire. Pour occuper la jeunesse à la fin des classes, il la met à l'apprentissage de la musique : " je me trouve mieux de les entendre racler sur leurs violons, que d'avoir la tête paralysée des bruits horribles qu'ils faisaient, sans compter les avantages 
qu'ils en retireront $" .{ }^{29}$ L'indiscipline était-elle aussi grande que le disait le nouveau directeur? Au vrai, il est difficile de le préciser, car les sources livrent bien peu de détails sur le déroulement concret des journées lors des premières années d'existence du collège. Chose certaine, le règlement initial avait de quoi choquer n’importe quel ecclésiastique habitué à un régime de vie nettement plus strict. Il est possible, au demeurant, que Proulx ait noirci un peu le portrait pour donner une image favorable de ses propres réalisations. Quoi qu'il en soit, malgré le redressement disciplinaire, la souplesse et une certaine indulgence semblent continuer de caractériser la conduite de la maison, ce qui suscite régulièrement des critiques et de l'inquiétude à l'évêché de Québec. Même si on ne le clame pas dans les règlements, les élèves protestants peuvent encore bénéficier de mesures d'exemption pour l'enseignement religieux. ${ }^{30}$ Les principes en vigueur choquèrent d'ailleurs Alexis Mailloux, le rigoriste directeur qui succéda à Proulx en 1834 .

\section{«Une éducation propre à la vie matérielle de la société " ${ }^{31}$}

Les années pendant lesquelles Proulx puis Mailloux dirigent le collège sont marquées par de nombreuses difficultés. La pénurie de personnel enseignant est un problème récurrent. Painchaud doit multiplier les lettres à son évêque pour le convaincre d'envoyer à Sainte-Anne quelques régents qui pourront faire la classe tout en poursuivant leurs études théologiques. Le recrutement des élèves suit par ailleurs un mouvement en dents-de-scie, et la santé financière du collège est des plus précaires. Comme bon nombre de parents n'arrivent pas à payer la pension annuelle, l'établissement doit consentir des réductions s'il veut conserver sa clientèle. Il lui faut aussi chercher le soutien pécuniaire de la notabilité locale et de la Chambre d'Assemblée.

Linstitution n'en poursuit pas moins un triple objectif : offrir un cours d'étude classique de huit années, faire de l'éducation civique et procurer aux élèves divers savoir-faire qui, en plus de les divertir entre les classes, pourront leur être utiles dans leur vie adulte. Le premier objectif est difficilement atteint au cours de ces premières années et, à quelques reprises, les directeurs de la maison s'en plaignent. Pour atteindre le second, le programme prévoit interroger les élèves "sur les affaires du tems, qu'ils doivent connaître par la lecture des papiers publics ${ }^{32}$ Quant au dernier, il se réalise par des leçons pratiques visant à initier les collégiens au dessin et à la sténographie. Ceux qui le désirent peuvent aussi occuper leurs temps libres au travail du bois ou du fer. En 1833, Painchaud conçoit l'idée de donner un bain linguistique en anglais aux plus talentueux de ses élèves. Il s'adresse à cette fin à l'abbé Deluol, supérieur du séminaire sulpicien de Baltimore, pour lui proposer la mise sur pied d'un programme d'échanges entre les deux institutions. ${ }^{33}$ Aucune trace de la réponse du sulpicien, si réponse il y eut, n’a cependant été retrouvée dans les archives.

Si le projet de favoriser l'immersion linguistique ne se concrétise pas en 1833, celui d'offrir aux jeunes ruraux du Bas-Saint-Laurent un enseignement plus poussé en anglais, allant au-delà des quelques leçons et exercices hebdomadaires inscrits au programme, n'est pas abandonné. Il est même relancé en 1840 , après la mort de Painchaud. Au mois d'août, à l'issue des examens publics de fin d'année, quelques 
notables de la région et l'abbé Mailloux, alors supérieur du collège et curé de SainteAnne, lancent une souscription pour introduire " un cours abrégé et séparé, purement anglais " et pour financer la construction d'un édifice destiné à accueillir les nouveaux élèves. ${ }^{34}$ Avec ce projet de cours commercial en quelque sorte, les autorités collégiales et les laïcs qui les appuient — comme les marchands Dionne et Casgrain — s'efforcent résolument de s'adapter aux caractéristiques et aux besoins du milieu. Pragmatiques, ils ont compris que la survie de l'institution nécessitait quelques innovations, et qu'en dépit du prestige dont on s'efforçait de les revêtir, les humanités n'étaient guère un facteur attractif suffisant pour un collège situé en plein cœur d'une région agricole. Ils imaginent donc un programme de formation pratique et accessible "à la majorité des habitants [des] campagnes, incapables de soutenir les frais d'un cours de haute éducation ». ${ }^{35}$ Par ailleurs, l'acquisition d'une bonne connaissance de l'anglais est perçue comme un avantage rapidement monnayable, dont l'attrait surpasse sans doute, aux yeux de plusieurs, le vernis de latin péniblement acquis au contact de Cicéron, de Salluste et de Virgile.

Le cours commercial, aussi appelé " cours anglais " ou " cours français-anglais ", ouvre ses portes en 1842. Il s'inspire d'un programme offert par l'Académie du collège jésuite de Georgetown qui, depuis sa fondation en 1786, cherche à procurer à " toutes les classes de citoyens » un enseignement pratique et à préparer ceux qui le voudront à l'enseignement classique. ${ }^{36}$ À Sainte-Anne, il n'est donc plus question de méthodes lancastriennes et de comités d'élèves élus par leurs pairs. L'organisation de la maison s'aligne désormais sur les principes disciplinaires et pédagogiques observés ailleurs. Les innovations se trouvent plutôt du côté du programme et des objectifs qu'il poursuit. À ses débuts, le cours commercial du Collège de Sainte-Anne compte trois niveaux - un quatrième est bientôt ajouté — et les élèves peuvent y être admis dès l'âge de 9 ans. Le prospectus, rédigé par le premier préfet des études, ThomasBenjamin Pelletier, et publié dans les journaux de l'époque, indique les matières à l'étude. La première année est consacrée à la grammaire française et anglaise, à l'arithmétique et la calligraphie. À son terme, les élèves sont censés savoir lire et écrire correctement l'anglais. La géographie, l'histoire, la tenue de livres et le dessin technique s'ajoutent à ces matières, la deuxième année. Enfin, la troisième et dernière année comporte, en plus des matières précédemment énumérées, des cours de mathématiques, de géométrie pratique, de mécanique et d'architecture (en anglais), d'astronomie (pour le cultivateur, précise le programme), de botanique et de minéralogie. ${ }^{37} \mathrm{Au}$ terme de ce cursus, il est possible, pour les élèves, "si leur fortune $\&$ leurs talens les rendent capables de profiter d'une éducation plus complète ", de s'inscrire à un cours classique présentant quelques différences avec le programme jésuite. ${ }^{38}$ Dans les sollicitations qu'ils adressent au clergé afin d'attirer des dons, les dirigeants du collège font aussi valoir la nécessité pour l'Église d'occuper rapidement le champ de l'« éducation secondaire "— qu'il distingue des « études classiques " — au moment où le gouvernement, avec la loi scolaire de 1841, cherche à relancer l'instruction primaire :

Si les catholiques canadiens, avertis de ce qui vient de se passer au sujet de l'éducation primaire, ne se hâtent point, de concert avec le clergé, de soustraire 
une partie de notre jeunesse à l'influence morale et religieuse qu'une tendance ennemie, venant de divers points à la fois, semble décidée à lui imprimer, le temps viendra vite où la religion et tous les intérêts canadiens ne seront plus qu'un triste objet de regrets inutiles et d'amers repentirs. ${ }^{39}$

Comme en 1830, au temps de l'abbé Chartier, le projet éducatif de Sainte-Anne prend donc une coloration nationale. Investir l'enseignement commercial, c'est tout à la fois faire œuvre patriotique et religieuse, et travailler à consolider l'influence de l'Église. En plus de préparer à la vie professionnelle, les différentes activités pédagogiques et extra-pédagogiques qui meublent le quotidien poursuivent l'objectif d'instiller les vertus morales, de propager les dévotions et de faire connaître les fondements du catholicisme.

Avec l'implantation du cours commercial, le collège prend un virage important. Il revoit notamment toute l'organisation du cours classique. Le programme des classes d'éléments latins, de syntaxe et de méthode est concentré en une seule année. Par la suite, les élèves font la versification, les belles-lettres, la rhétorique et, s'ils le souhaitent, les deux années de philosophie. À moins d'avoir commencé leurs études dans une autre institution, les collégiens de Sainte-Anne passent donc obligatoirement par le cours commercial où l'enseignement se fait pour une bonne part en anglais. L'expérience, soulignons-le, n'est pas tout à fait nouvelle dans le paysage scolaire québécois, le Collège de Montréal ayant offert de 1789 à 1830, dans le cadre de «l'école anglaise ", un cours commercial fréquenté par des élèves de 7 ans à 30 ans..$^{40}$ Avec la mise en place du programme de Sainte-Anne, la filière commerciale semble toutefois connaître un nouveau départ. Au milieu du $19^{\mathrm{e}}$ siècle, d'autres établissements collégiaux décident d'élargir eux aussi leur offre éducative en proposant un cursus semblable à celui de La Pocatière. ${ }^{41}$ Selon Jean-Pierre Charland, 15 des 19 " collèges classiques " de la fin du $19^{\mathrm{e}}$ siècle disposent d'un cours commercial. ${ }^{42}$ Dans la première moitié du $20^{\mathrm{e}}$ siècle, ces établissements, à quelques exceptions près, abandonnent toutefois cette filière aux frères enseignants et se limitent aux seules humanités. ${ }^{43}$

Le programme commercial du collège pocatois connaît pour sa part une pérennité certaine. En dépit des efforts déployés par les autorités diocésaines pour promouvoir le cours classique, au détriment, parfois, de la filière commerciale, il perdure pendant un siècle. Au cours des années, ce profil fait l'objet d'un certain nombre de modifications. Les manuels sont périodiquement renouvelés et de nouvelles matières obligatoires ou facultatives - entre autres le droit civil et commercial, la dactylo, la sténographie ou la télégraphie — sont ajoutées. ${ }^{44}$ Les derniers élèves à s'inscrire à ce cursus font leur entrée en 1943. À partir de cette date, le collège abandonne graduellement l'enseignement commercial, qui disparaît complètement en 1946, alors que les pressions épiscopales se font de plus en plus fortes pour bien distinguer les établissements classiques des institutions offrant le cours commercial ou technique. Plus de 100 ans après sa fondation, il devient véritablement et complètement un " collège classique ». Les jeunes désirant obtenir une formation commerciale devront dorénavant aller étudier dans les établissements spécialisés de la région, notamment à Saint-Pacôme, à L'Islet, à Montmagny ou à Rivière-du-Loup. 


\section{Le cours commercial : porteur d'une croissance soutenue du Collège}

En accord avec la culture marchande et technique alors en plein essor, le tournant opéré au cours de la décennie 1840 reflète une conception utilitaire de l'éducation. Habiles, les promoteurs de ce programme ont su lui gagner l'appui de plusieurs clercs et de laïcs exerçant leurs activités et leur influence au-delà de la sphère régionale. ${ }^{45}$ C'est par exemple le cas du chanoine Hyacinthe Hudon de Montréal, du gouverneur Charles Bagot et du notaire Edouard Glackemeyer, de Québec. Plus tard, à la fin du $19^{\mathrm{e}}$ siècle, Louis Fréchette, contempteur des études classiques, citera en exemple le programme du Collège de Sainte-Anne, qui " contient d'excellentes choses ". L'on y découvre, ajoute-t-il, " une tendance bien prononcée à briser avec les vieilles routines $" .46$

La nouvelle filière, qui offre une alternative au cours classique, semble répondre aux besoins et aux attentes de nombreux parents désireux d'offrir à leurs fils une instruction susceptible de leur permettre de s'inscrire dans l'économie marchande. L'institution recrute l'essentiel de ses élèves dans les comtés ruraux de la Côte-duSud et du Bas-du-Fleuve. Des élèves de Charlevoix, du Nouveau-Brunswick et de la Beauce la fréquentent aussi. Une soixantaine de jeunes s'y inscrivent la première année. Dans la décennie qui suit, le nombre de nouvelles admissions est un peu moins spectaculaire : certaines années, elles dépassent la cinquantaine, d'autres années, elles se situent aux alentours de 25 ou 30. En dépit de ces fluctuations, le virage commercial a de toute évidence été un choix porteur pour l'institution, à défaut d'être rentable financièrement, du moins à ses débuts. ${ }^{47}$ Une centaine d'élèves la fréquentent en 1843. Ce nombre grimpe à 247 en 1858 . Jusqu'au tournant du $20^{\mathrm{e}}$ siècle, la population étudiante oscille généralement entre 230 et 275 , et dépasse même 300 personnes en 1900. Le bâtiment a alors atteint sa capacité maximale d'accueil. L'ouverture d'une nouvelle aile, en 1901, puis d'une autre en 1918, et la reconstruction du collège après l'incendie de 1921 conferent à l'établissement davantage d'espace qu'une clientèle grandissante viendra bientôt occuper. Vers 1930, environ 600 élèves, dont une majorité de pensionnaires, étudient à Sainte-Anne.

Quelle est la part du cours commercial dans cette augmentation quasi continue d'élèves? Les annuaires du Collège, publiés à partir de 1888, sont ici d'un précieux secours. Ils indiquent avec précision le nombre d'élèves inscrits dans chacune des options, classique et commerciale, de la fin du $19^{\mathrm{e}}$ siècle jusqu'à la fermeture définitive de la seconde en 1946. Au tournant du $20^{\mathrm{e}}$ siècle, le cours commercial est sans conteste le plus fréquenté des deux cursus (voir Graphique). Il regroupe à cette époque $56 \%$ à $68 \%$ de la clientèle totale de Sainte-Anne. Pour une majorité, ce cours est terminal. Selon les statistiques compilées par l'institution, 59\% des 6318 élèves ayant étudié au collège entre 1829 et 1926 ne fréquentèrent que le cours commercial, la majorité d'entre eux $(74 \%)$ pendant moins de quatre ans, le temps de savoir lire et écrire le français et un peu d'anglais et d'avoir acquis quelques notions d'arithmétique, de tenue de livres et de dessin. Près de la moitié (47\%) des élèves qui commencèrent leurs humanités interrompirent leurs études avant la Rhétorique. ${ }^{48}$ Seulement $15 \%$ de la clientèle du collège pocatois complétèrent l'ensemble du cours classique et 11,8\% 
devinrent prêtres ou religieux. À cet égard, l'exemple de Sainte-Anne invite à nuancer fortement l'image d'Épinal des collèges, pépinières de prêtres et de religieux. D'après les chiffres fournis pour commémorer le $100^{\mathrm{e}}$ anniversaire de fondation, l'institution a fourni entre 1829 et 1926 davantage de marchands et de commis que de prêtres.

\section{Élèves du cours classique et du cours commercial au Collège de Sainte-Anne, 1887-1950}

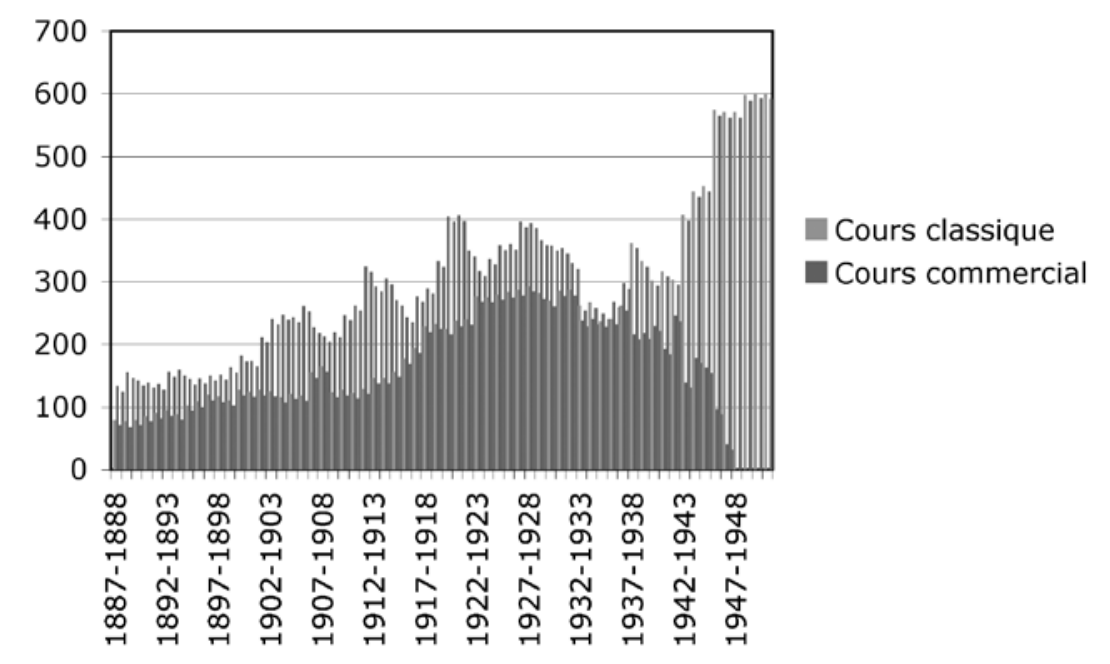

En guise de conclusion : le long débat sur la meilleure éducation... et son issue

Bien qu'il suscite l'enthousiasme et qu'il inspire d'autres collèges, le cours commercial de Sainte-Anne provoque aussi malaise et critiques parmi les milieux ecclésiastiques. À Québec, au sein du séminaire comme à l'évêché, il s'en trouve plus d'un qui regarde avec mépris le projet éducatif du petit collège de campagne. En témoigne ce brouillon d'une lettre de 1859 conservé aux archives :

Il paraît donc que les MM. du Séminaire nous laissent à débrouiller la matière et gardent pour eux l'intelligence, encore croient-ils nous honorer beaucoup en nous faisant cette modeste part. Il faut avouer que notre cours français anglais leur fait mal au cœur, 160 à 180 élèves de plus dans une maison comme la nôtre donnent une forte recommandation et c'est une preuve évidente que le pays comprend l'importance d'une telle instruction.

Quant au système qui répond le mieux au besoin moral, c'est-à-dire au plus grand développement intellectuel au pays, je croirais que c'est celui qui 
donnerait la plus grande somme de connaissances sur toute espèce de sciences utiles. On dirait, que selon ces MM. le grec et le latin ont le monopole de l'intelligence et que ceux qui n'en savent rien, ne peuvent aspirer à l'intelligence. Je pense que l'élève peut exercer son intelligence aussi bien en traduisant de l'anglais en français que du grec et du latin. Il en retire le même profit pour sa langue qu'il étudie non pas six ans, mais 7 à 8 ans. Mais dirait-on, on ne parle pas à la parisienne, donc on perd sa nationalité, son patriotisme, on n'écrit pas le français comme l'abeille, donc on est ignorant; de grâce, je vous en prie, c'est avoir un peu trop de prétention que de vouloir faire admirer ce qui est insipide et bien peu en harmonie avec des intelligences si sublimes. ${ }^{49}$

On trouve ici les principaux éléments de la controverse appelée à se poursuivre pendant plusieurs décennies. Elle oppose les tenants d'une éducation utilitariste et pratique préparant au monde du travail et ceux prônant une formation désintéressée fondée largement sur l'apprentissage des humanités. Comme le révèlent les projets développés à La Pocatière, la position du clergé sur cette question n’est pas univoque. Loin d'être l'apanage de la bourgeoisie libérale ${ }^{50}$, le souci d'un enseignement cherchant à favoriser l'insertion sociale et économique par l'acquisition de divers savoirfaire s'est manifesté au sein même de l'Église et a rencontré l'adhésion d'une partie de la population rurale, ce qui laisse entrevoir l'influence du milieu dans la définition de l'offre éducative. Ainsi, dès le début du $19^{\mathrm{e}}$ siècle, certains prêtres ont voulu proposer des programmes d'études et des modèles pédagogiques s'éloignant peu ou prou de la Ratio jésuite. Ils ont, à cette fin, intégré avec plus ou mois de bonheur les propositions des réformateurs européens des $18^{\mathrm{e}}$ et $19^{\mathrm{e}}$ siècles. Certes, les collèges enseignaient les humanités, et leur personnel enseignant croyait aux vertus du latin et des grands auteurs classiques pour élever le cœur, l'âme et l'intelligence de l'élève, mais un certain nombre de ces établissements faisaient aussi la part belle à d'autres matières. Dans le cas du Collège de Sainte-Anne, diverses raisons où s'entremêlent des facteurs financiers, le désir de répondre aux attentes des familles rurales et l'objectif de permettre à l'Église d'occuper le champ de l'éducation, ont conduit à rompre avec la "tradition " en proposant, à partir des années 1840, un cursus comparable aux enseignements des écoles techniques et commerciales canadiennes, américaines et européennes. ${ }^{51}$ Malgré ses caractères originaux, Sainte-Anne de La Pocatière n'est pas unique. D'autres collèges « classiques " offraient aussi des parcours commerciaux ou industriels, par exemple Joliette, Sherbrooke, Saint-Hyacinthe et Sainte-Marie de Monnoir, pour n'en nommer que quelques-uns. L'attrait qu'ils suscitent auprès de la population contredit cette idée d'un " atavisme » de la paysannerie et de la bourgeoisie canadienne-française marquées d'une vision du monde profondément " ruraliste " et opposée au commerce. ${ }^{52}$

La popularité et la longévité du cours commercial de La Pocatière invitent à s'interroger sur ce qu'ont été réellement les collèges dit " classiques " pendant une bonne partie de leur histoire. Loin de se restreindre à l'enseignement des humanités, des collèges pour garçons, comme Sainte-Anne, ont offert à nombre d'élèves une initiation aux matières dites commerciales, censées leur ouvrir les portes des milieux de la 
fabrication, des affaires et de la finance. Les collèges, du moins certains d'entre eux, n'auraient donc pas été, au $19^{\mathrm{e}}$ siècle, ces milieux routiniers, ployant sous le poids de la tradition, que plusieurs ont décrits.

$\mathrm{Au}$ vrai, l'histoire de ces établissements fournit un vaste champ d'investigation, où beaucoup reste encore à faire pour connaître finement les enseignements comme l'organisation des maisons, notamment en vue de préciser les facteurs et les enjeux de pouvoir ayant mené à l'abandon des filières commerciales à la fin du $19^{\mathrm{e}}$ siècle et au $20^{\mathrm{e}}$ siècle. Comme nous l'avons vu, à Sainte-Anne, le repli sur le cours classique s'est produit à une époque relativement tardive. Il a suscité des tensions entre les promoteurs de ce changement, notamment l'archevêque de Québec, désireux de spécialiser les institutions d'enseignement et de différencier les clientèles et leurs maîtres, et une partie du clergé enseignant, aspirant, pour des considérations financières ou pour d'autres motifs, à conserver le cours commercial. Sainte-Anne est-il un des derniers collèges, avec Mont-Laurier et Sherbrooke, ${ }^{53}$ à avoir offert la double filière? Il est possible à ce chapitre que les années 1940 et 1950 aient marqué un moment charnière dans cette transformation de l'institution, celle d'un recentrage définitif autour des humanités.

L'exemple de Sainte-Anne soulève aussi des questions sur l'influence qu'ont eue sur ceux qui les ont fréquentés les collèges pour garçons. Qu’a signifié la vie dans cette institution pour cette majorité d'élèves ayant abandonné les études avant même d'avoir ouvert leur première grammaire latine ? Les témoignages laissés par les anciens mettent largement l'accent sur la rigueur de la discipline, l'émulation comme pratique pédagogique, les contraintes (ou les joies) de la vie en groupe et la prégnance du religieux. ${ }^{54}$ Beaucoup auront sans doute été davantage marqués par ces aspects de la vie quotidienne que par les grands auteurs classiques ou les versions latines. Pour les élèves qui connurent l'expérience du pensionnat, mais aussi fort probablement, pour leurs confrères externes, les années au collège auront signifié réglage du temps, ordonnancement de l'espace, dressage quotidien du corps dans un moule, le collègepensionnat, auquel les familles donnaient largement leur adhésion. ${ }^{55}$ C'est peut-être de ce côté, dans cet apprentissage de la vie sociale, de règles comportementales, de méthodes de travail, d'un rapport à soi, à l'autre, à la connaissance, qu'il faut rechercher l'héritage commun qu'ont laissé les collèges pour garçons, du $19^{\mathrm{e}}$ siècle au milieu du siècle suivant.

\section{Notes}

* La traduction du résumé est de Jean-François Duranleau et de Jean-Philippe Guertin, sous la supervision de leur professeure d'anglais, Diane Pigeon.

1 Claude Galarneau, Les collèges classiques au Canada français (1620-1970) (Montréal : Fides, 1978), 8.

2 Le contraste entre les deux projets éducatifs est bien formulé dans Paul Inchaupsé, «Un nouvel humanisme, socle du nouveau système d'éducation proposé ", Bulletin d'histoire politique, Le Rapport Parent, 1963-2003, 12, 2 (hiver 2004) : 66-80. 
3 Galarneau, Les collèges classiques, 242.

4 Ibid., 151.

5 Sur l'histoire socio-économique de la région, voir Alain Laberge, dir., Histoire de la Côtedu-Sud (Québec : IQRC, 1993), 71-123, 153-172 en particulier.

6 Pour reprendre l'expression d'Alain Laberge, ibid., 153.

7 Voir notamment Inchaupsé, «Un nouvel humanisme. Mentionnons également ici : les deux synthèses d'Andrée Dufour, Tous à l'école. État, communautés rurales et scolarisation au Québec de 1826 à 1859 (Montréal : Éditions Hurtubise HMH, 1996) et Histoire de l'éducation au Québec (Montréal : Éditions du Boréal Express, c2004); et celles de Jean-Pierre Charland, L'entreprise éducative au Québec, Québec, 1840-1900 (SainteFoy : Les Presses de l'Université Laval, 2000) et Histoire de l'éducation au Québec. De l'ombre du clocher à l'économie du savoir (Saint-Laurent : ERPI, 2005). Dans une tout autre perspective, le groupe de recherche Hermès a notamment mis en lumière les transformations qu'a connues l'enseignement de la rhétorique au Québec entre 1760 et 1840. Voir les travaux du groupe sur http://www.unites.uqam.ca/arche/hermes/

8 Serge Gagnon, "Painchaud, Charles-François ", Dictionnaire biographique du Canada en ligne, http://www.biographi.ca/FR. Centre d'archives de la Côte-du-Sud et du Collège de Sainte-Anne (CASA), Fonds Collège de Sainte-Anne, Supériorat de Painchaud.

9 Écoles promues par l'éducateur anglais Joseph Lancaster (1778-1838). Dans ses classes, Lancaster avait instauré un système de monitorat où les élèves plus âgés et plus avancés assuraient l'instruction des plus jeunes. Voir Heather Lysons-Balcon, « Lancaster, Joseph ", Dictionnaire biographique du Canada en ligne, http://www.biographi.ca/FR.

10 Yvan Lamonde, "Classes sociales, classes scolaires ", Sessions d'études, Société canadienne d'histoire de l'Église catholique, 41 (1974) : 43-59.

11 CASA, Fonds Collège de Sainte-Anne, Prospectus. Voir aussi dans le même fonds d'archives le Manifeste de Painchaud, $1^{\text {er }}$ août 1828.

12 Voir Louis-Philippe Audet, Histoire de l'enseignement au Québec, t. 1, 1608-1840 (Montréal/Toronto : Holt, Rinehart et Winston, 1971), 365-368 et Dufour, dans Histoire de l'éducation au Québec, 24, notamment. L'enseignement mutuel fut surtout pratiqué au niveau élémentaire au $19^{\mathrm{e}}$ siècle. Pour la France, voir Françoise Mayeur, De la Révolution à l'École républicaine (1789-1930), Tome III d'Histoire générale de l'enseignement et de l'éducation en France dirigé par Louis-Henri Parias (Paris : Nouvelle Librairie de France, 1981), 372-389.

13 CASA, Fonds Collège de Sainte-Anne, Supériorat Charles-François Painchaud, État du collège, $1^{\text {er }}$ août 1828 .

14 Ibid.

15 CASA, Fonds Collège de Sainte-Anne, Programmes d'études, Plan d'éducation, 1827.

16 Wilfrid Lebon, Histoire du collège de Sainte-Anne-De-La-Pocatière. Le premier demi-siècle 1827-1877 (Québec : Charrier \& Dugal, 1948), 10 et 413.

17 CASA, Fonds Collège de Sainte-Anne, Programmes d'études, Plan raisonné d'un cours d'études pour le Collège de Sainte-Anne, 17 novembre 1828. Sur Chartier, on lira avec profit la biographie de Richard Chabot, "Chartier, Étienne ", Dictionnaire biographique du Canada en ligne. http://www.biographi.ca/FR.

18 CASA, Fonds Collège de Sainte-Anne, Programmes d'études, Plan raisonné d'un cours d'études pour le Collège de Sainte-Anne, 17 novembre 1828. Dans son livre, Claude Galarneau évoque d'ailleurs les débats autour de l'enseignement classique, notamment celui qui prend place dans les pages de la Minerve en 1829. Galarneau, Les collèges classiques, 219-220.

19 CASA, Fonds Collège de Sainte-Anne, Programmes d'études, Plan raisonné d'un cours d'études pour le Collège Sainte-Anne, 17 novembre 1828.

20 Marcel Grandière, Lidéal pédagogique en France au XVIII siècle (Oxford : Voltaire Foundation, 1998), 61. 
21 CASA, Fonds Collège de Sainte-Anne, Programmes d'études, Plan raisonné d'un cours d'études pour le Collège de Sainte-Anne, 17 novembre 1828.

22 L'exemple de Sainte-Anne est ici bien mal choisi pour montrer le caractère autoritaire des collèges créés au début du $19^{\mathrm{e}}$ siècle. Fernand Ouellet, Le Bas-Canada 1791-1840. Changements structuraux et crise (Ottawa : Éditions de l'Université d'Ottawa, 1976), 109.

23 Comme l'a montré Ollivier Hubert, la proportion d'élèves externes est à peu près équivalente au Collège de Montréal à la même époque.

24 CASA, Fonds Collège de Sainte-Anne, Supériorat Charles-François Painchaud, C.S. Cherrier à Painchaud, 19 septembre 1831.

25 Lebon, Histoire du collège de Sainte-Anne.

26 Chartier sera par la suite curé de Sainte-Martine-de-Châteauguay, de Saint-Pierreles-Becquets, de Saint-Patrice (Rivière-du-Loup), puis de Saint-Benoît à compter de 1835. Il participe alors aux assemblées des patriotes. En 1837, il prononce quelques sermons par lesquels il encourage ses paroissiens à prendre les armes et, en 1838, il se range momentanément aux côtés de Nelson, avec lequel, cependant, il connaîtra assez rapidement des dissensions. Voir à ce propos Richard Chabot, Le curé de campagne et la contestation locale au Québec de 1791 aux troubles de 1837-38 (Montréal : Hurtubise MHM, 1975), 117-121.

27 Chabot, "Chartier, Étienne".

28 CASA, Fonds Collège de Sainte-Anne, Lettre de Louis Proulx à Charles Cazeau, 19 octobre 1830.

29 CASA, Fonds Collège de Sainte-Anne, correspondance expédiée, boîte 249, dossier n ${ }^{\circ}$ 19, lettre de Louis Proulx à Charles Cazeau, 23 janvier 1831.

30 Comme le révèle la correspondance du collège, où le directeur promet à quelques pères protestants d'exempter leurs fils de l'assistance à la messe.

31 CASA, Fonds Collège de Sainte-Anne, Supériorat François Pilote, Appel au clergé, 19 mars 1842.

32 CASA, Fonds Collège de Sainte-Anne, Institution, Programme d'études, Enseignement au collège de Sainte-Anne, 21 juillet 1831.

33 N.-E. Dionne, Vie de C.-F. Painchaud prêtre, curé, fondateur du collège de Sainte-Anne de la Pocatière (Québec: Brousseau, 1894).

34 CASA, Fonds Collège de Sainte-Anne, "Aux amis de l'éducation ", Souscription en faveur du Collège de Sainte-Anne, 18 août 1840.

35 Ibid. L'importance accordée à l'apprentissage d'une seconde langue vivante distingue la société bas-canadienne de bien d'autres milieux où le besoin d'offrir un tel enseignement ne se pose pas, ou se pose peu.

36 Comme l'indique un document du collège : CASA, Fonds Collège de SainteAnne, Institution, Programme d'études, Plan du nouveau cours d'études, 4 juin 1842; " Proposals for Establishing an Academy at George-town, Patowmack-River, Maryland ", novembre 1786, reproduit dans Paul R. O. Neill and Paul K. Williams, The College History Series, Georgetown University (Charleston : Arcadia Publishing, 2003), 11. L'influence de Georgetown se fait aussi sentir à Saint-Hyacinthe, mais de manière différente. Après les études qu’il y effectue, l'abbé Isaac-Stanislas Désaulniers développe l'enseignement des sciences à Saint-Hyacinthe. C.-P. Choquette, Histoire du Séminaire de Saint-Hyacinthe depuis sa fondation jusquà nos jours, tome 1 (Montréal : Imprimerie de l'institution des sourds-muets, 1991), 152.

37 CASA, Fonds Collège de Sainte-Anne, Institution, Programme d'études, Plan du nouveau cours d'études, 4 juin 1842 . Un prospectus en anglais est aussi rédigé : Prospectus of the course of English education to be introduced in the new College of Ste Ann's La Pocatière. 
38 CASA, Fonds Collège de Sainte-Anne, Institution, Programme d'études, Plan du nouveau cours d'études, 4 juin 1842.

39 CASA, Fonds Collège de Sainte-Anne, Institution, Programme d'études, Appel au clergé, 19 mars 1842.

40 Ollivier Hubert, "Petites écoles et collèges sulpiciens » dans Dominique Deslandres, John Dickinson et Ollivier Hubert, Les Sulpiciens de Montréal. Une histoire de pouvoir et de discrétion 1657-2007 (Montréal : Fides, 2007), 414-415.

41 Ainsi, en est-il, par exemple, du Collège de Joliette, de celui de Sainte-Marie-deMonnoir et, à compter de 1875, du Collège Saint-Charles-Borromée de Sherbrooke. Les jésuites eux-mêmes tentent une première expérience du cru à Montréal, au Collège Saint-Marie, en 1849. Ils abandonnent toutefois l'option commerciale en 1856, la réactivent en 1866, avant de la fermer définitivement en 1888. Jean Cinq-Mars, Histoire du collège Sainte-Marie de Montréal, 1848-1969 (Montréal : HMH, 1998), 92-93.

42 Charland, L'entreprise éducative au Québec, 251.

43 Félix Bouvier a étudié l'un de ces collèges offrant les deux cursus dans son Histoire $d u$ Séminaire de Mont-Laurier. Formation d'une élite et d'une classe moyenne (Montréal : Fides, 2005), 216 p.

44 Annuaires du collège de Sainte-Anne.

45 Voir à ce propos Serge Gagnon, "Le clergé, les notables et l'enseignement privé au Québec : le cas du collège de Sainte-Anne, 1840-1870 ", Histoire sociale/Social History 3, 5 (avril 1970) : 45-65.

46 Louis Fréchette, À propos d'éducation. Lettres à M. l'abbé Baillargé du collège de Joliette (Montréal : Désaulniers, 1893), 91.

47 L'institution éprouve des difficultés financières importantes pendant une bonne partie du $19^{\mathrm{e}}$ siècle. Voir Serge Gagnon, "Pilote, François, Dictionnaire biographique du Canada en ligne, http://www.biographi.ca/FR/.

48 Catalogue des anciens élèves du collège de Sainte-Anne-de-la-Pocatière, 1827-1927 (Québec : L’Action sociale, 1927).

49 F100, Fonds Collège de Sainte-Anne, correspondance reçue, boîte 238, dossier no 16, lettre d'Amable Blanchet (prêtre enseignant à Sainte-Anne) à un destinateur inconnu,

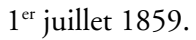

50 Plusieurs études ont souligné les critiques formulées par les libéraux à propos du cours classique et du clergé enseignant. Voir notamment Jean-Paul Bernard, Les Rouges. Libéralisme, nationalisme et anticléricalisme au milieu du XIXe siècle (Montréal : Les Presses de l'Université du Québec, 1971). On trouvera des exemples probants des reproches adressés au clergé dans Le Pays du 28 juillet, 28 août, 26 septembre 1855 et du 10 octobre 1855.

51 Voir par exemple D. K. Müller, Fritz K. Ringer, B. Simon, dir., The Rise of the Modern Educational System. Structural Change and Social Reproduction, 1870-1920 (Cambridge : University Press/Maison des Sciences de l'Homme, 1989).

52 Ouellet, Changements structuraux et crise, 109-118 notamment.

53 Sherbrooke abandonne le cours commercial en 1948, Mont-Laurier en 1952. Bouvier, Histoire du Séminaire de Mont-Laurier. Sur Sherbrooke, lire http://www.seminairesherbrooke.qc.ca/index.php? module=CMS\&func=view\&id $=41$.

54 Claude Corbo, La mémoire du cours classique. 1. Les années aigres-douces des récits autobiographiques (Montréal : Éditions Logiques, 2000).

55 Voir à ce propos Bernard Delpal et Olivier Faure, dir., Religion et enfermements $\left(X V I I^{e}-X X^{e}\right.$ siècles) (Rennes : Presses universitaires de Rennes, 2005). 